\title{
Philippine Welser als Freundin der Heilkunst
}

\author{
Von Karl BeER, Wien
} Unzweifelhaft zählt die wegen ihrer Schönheit gefeierte Augsburger Patrizier-
tochter PHILIPPINe Welser zu den bedeutendsten Frauengestalten, denen wir
im 16. Jahrhundert an den europäischen Fürstenhöfen begegnen. Ein Nachfahre
aus dem Geschlechte der WELSER meinte, daß PHILIPPINEs Lebensverhältnisse
in der Geschichte einzig dastünden. Es ist daher begreiflich, daß sich Dichtung
und Geschichtsforschung ihrer Persönlichkeit immer wieder zuwandten.

Nicht alle Rätsel, die sich an den Lebenslauf Philippines knüpfen, sind heute schon gelöst. Die historische U̇berlieferung ist zu karg zugemessen. Das liegt in der Tatsache mitbegründet, daß Philippine 1557 dem Habsburger Erzherzog FerDINAND, der damals Statthalter von Böhmen war, heimlich angetraut wurde und daß von den folgenden 23 Jahren ehelicher Verbundenheit nicht weniger als 19 der Geheimhaltung unterworfen blieben. Damit war begreiflicherweise auch der Nährboden bereitet, auf dem Erdichtungen und Gerüchte gedeihen mochten. Zur Geheimhaltung ihrer Ehe «zu ewigen Zeiten» hatte sich das Paar verpflichtet, als der kaiserliche Vater für den gegen seinen Willen und ohne sein Wissen getanen Schritt Verzeihung gewährte. Die Verschwiegenheit mußte weiterbestehen, so sehr sie die Betroffenen bedrücken mochte, bis 1576 der Papst das Ehepaar von dem harten Eide entband.

Die langen Jahre nicht offenen ehelichen Lebens, erst auf Schlössern im mittleren Böhmen, hernach auf Schloß Ambras in Tirol verbracht, waren für PHILIPPINE Jahre der Zurückgezogenheit, aber gleichwohl auch Jahre familiären Glücks. Bei Erzherzog Ferdinand hatte sich ein Wandel vollzogen, er, der bis zu dem Zeitpunkte, da er die tiefste Neigung zu Phippine faßte, keine strengere Lebensführung gekannt hatte, blieb seiner Gemahlin völlig zugetan und sein Hof galt als Stätte guter Sitte, solange Philippine an seiner Seite weilte. Philippine schenkte ihrem Gatten vier Kinder. Zwei Knaben sind am Leben geblieben: Andreas, der als römischer Kardinal und Bischof, und KArL, der als Markgraf von Burgau in die Geschichte einging. Von der Nachfolge in der Regierung der österreichischen Erblande waren diese Prinzen bekanntlich ausgeschlossen.

Die Zuneigung aller habsburgischen Verwandten zu gewinnen, ist PhiLIPpine nicht gelungen, wäre wohl auch einem anderen Kinde gleicher Herkunft nicht gelungen. Philippines Schwager, Kaiser Maximilian II., spricht gelegentlich von einer Verzauberung seines Bruders FERdinand, herbeigeführt von einer «losen brekin», worunter kaum jemand anderer zu verstehen war als Philippine, und die Schwägerin Maria von der steirischen Linie brachte es über sich, PhiLIPPINES Söhne mit der lieblosen Bezeichnung «pannckharten» zu bedenken. 
Ferdinand hat seiner Gemahlin vielfache Beweise seiner Liebe und Wertschätzung gegeben. Als er Landesherr von Tirol wurde (1564), schenkte er ihr das Schloß Ambras, das sich unfern von Innsbruck im Mittelgebirge erhebt und beherrschend über das Inntal hinwegblickt. Die Übergabe geschah mit den ehrenden Widmungsworten: «Der Edlen Philippina Welserin sonderlich Irer ehrn und tugent wolverhaltens halben.»

Durch Um- und noch mehr durch Zubauten machte der kunstliebende Erzherzog die Residenz seiner Gemahlin, einen bis dahin wenig beachteten Edelsitz, zu einem der schönsten Schlösser in deutschen Landen und sicherte ihm durch sein rastloses, keine Auslagen scheuendes Sammeln von Waffen, Rüstungen, Gemälden, Büchern, Antiquitäten und Raritäten einen weithin reichenden Ruf. Im Jahre des Einzugs in Ambras (1567) ehrte der Erzherzog seinen Schwiegervater Franz Welser und dessen Nachkommen durch die Erhebung in den Freiherrnstand mit dem Prädikate von Zinnenburg. Dieses Prädikat führte auch Philippine.

Die lange Geheimhaltung der Ehe machte es verständlich, daß Philippine in der Öffentlichkeit nur wenig hervortrat. Erzherzog Frrdinand war echter Renaissancefürst. Er liebte nicht bloß die Kunst, sondern auch prunkvolle Festlichkeiten. Auf die Programmgestaltung nahm er jeweils entscheidenden Einfluß, übernahm selbst auch gerne bei der Durchführung eine führende Rolle. PhILIPPINE zog es in solchen Fällen vor, stille Zuschauerin zu sein. Wohl aber war sie unter den Mitwirkenden zu sehen, wenn es sich um kleinere Veranstaltungen handelte. Bei einem Armbrustschießen zeigte sie, daß sie den Pfeil so sicher zum Ziele zu senden verstand, wie sie sonst bei kunstvoller Stickerei die Nadel geschickt handhabte. Auch bei fröhlichen Besuchen in der Bacchusgrotte, wo der Erzherzog die Trinkfestigkeit seiner Gäste erproben und ihre Namen unter Beifügung eines launigen oder ernsten Sprüchleins im Trinkbuch verewigt wissen wollte, schloß sich Philippine nicht aus.

In die Politik des Landes mischte sich PhiLippine nicht ein, wohl aber übernahm sie es gerne, Fürsprecherin zu sein, wenn beim Landesherrn Bittgesuche eingelaufen waren. Im Laufe der Jahre hatten Tiroler aller Stände von der Schloßherrin zu Ambras Beweise der Teilnahme an ihrem Geschick und ihren Sorgen empfangen.

Noch schöneren Ausdruck fand Philippines soziales Empfinden in ihrer Betätigung als Krankenpflegerin.

Wie weit Philippines medizinisches Interesse reichte, das ist deutlich aus dem Rezeptbuch abzunehmen, das heute unter der Signatur 11.454 in der Nationalbibliothek zu Wien aufbewahrt wird. Die Handschrift in Folioformat zählt 130 Blätter mit Goldschnitt, 82 Seiten sind unbeschrieben. Die weitaus größere Zahl der Rezepte, es sind ihrer über zweihundert, ist von PHILIPPINe selbst geschrieben worden. Die Rezepte bezeichnete die Schreiberin nach der Sitte der Zeit gerne 
als «Kunst» oder auch als «gute Kunst», wenn die Arznei erfahrungsgemäß eine gute Wirkung getan hatte. Mit Bemerkungen am Schluß des Rezeptes, wie: «ist gut bewert», «ich habs probiert» oder «es sol helfen und gewiß sein», gibt PHILIPPINE einen Fingerzeig für kommende Benutzung.

Wie ist die Freundin häuslicher Heilkunst zu dieser Fülle von Rezepten gekommen? Fürs erste lieferten Beiträge die Hofärzte. Andere Rezepte gehen zurück auf den Augsburger Arzt Klaudius Peutinger und den hochgelehrten Herrn Bernhard Schludy, «beyder leyb und wundarzney docter, geschworner statartzat zu Augspurg». Diese Namen liefern unter anderem auch den Beweis dafür, wie sehr Philippine an ihrer alten Heimat hing. Andere Beiträge stammen von Philippines Base, Frau Katharina von Sternberg und deren Gemahl LAdislaus, dem Abkömmling eines bekannten böhmischen Adelsgeschlechts. Größer ist die Zahl der Rezepte, die meist adelige Frauen aus dem Lande Tirol zur Verfügung gestellt hatten. Da notierte Philippine einmal: «die forgeschribene stuck hat mir die fraw ScHwendin aus yrem biechly geschenkt, das hab ich abgeschrieben.» Andere Stücke «von der lieben mutter selligen» hebt Philippine pietätvoll hervor. Sechzehn Rezepte schließlich wurden der Fürstin von einem Juden überlassen.

Nach den knapp skizzierten Anweisungen sollten jeweils die Heilmittel hergestellt werden. Das waren Säfte, Getränke, Latwergen (eingedickte Säfte), Sirupe, Zeltchen, Pillen, Pulver, Salben, Pflaster und Pomaden.

Die Medikamentenbereitung fußte in weitaus überwiegendem Maße auf der Heranziehung von Heilpflanzen. Bei Bereitung eines «edlen guten Atem-Wassers» zur Bekämpfung der Atemnot kamen nicht weniger als achtzehn Pflanzen zur Verwendung. Man staunt über die große Zahl der in der Handschrift aufscheinenden Heilpflanzen und über die botanischen Kenntnisse der fürstlichen Frau. Jedenfalls sind diese Kenntnisse durch das Studium von Kräuterbüchern erworben worden, aber auch durch unmittelbare Beobachtung in der Natur selbst. Es war eine Auswirkung der Renaissancebewegung, daß man damals viel botanisierte und neuentdeckte Pflanzen mit Liebe beschrieb. Ein Beispiel der neuen Sammeltätigkeit bot der Leibarzt der Fürstin, Dr. Handsch. Er berichtet uns über die Heilpflanzen im Ambraser Schloßgarten wie in der Tiroler Bergwelt. An botanischen Anregungen für die Schloßherrin hat es also nicht gefehlt.

Geringer sind die Beiträge zur Arzneibereitung aus dem Tierreiche. Von kleinem Getier werden Schwalben und Schnecken verwendet. Der Krebs spendet seine Augen und Eier. Schmalz liefern der Hecht, Dachs und das Schwein, Unschlitt der Hirsch; Geile der Biber. Das Schaf wird einem Rezept zufolge in die Nähe des Kranken gebracht, geschlachtet und die rasch abgezogene Haut flugs auf den bloßen kranken Leib gelegt. Geschätzt sind die Klauen des Elentieres, die, in die Hände gelegt oder um den Hals gehängt, dem Kranken den 
Durst nehmen sollten. Ferdinands Agenten sind in Polen eifrigst bemüht, solche Klauen aufzutreiben.

Auch teure Steine werden gelegentlich zu Heilzwecken herangezogen.

Philippine stand mit ihrem starken medizinischen Interesse in ihrem Familienkreis nicht allein da. Auch ihr Gemahl sammelte Rezepte und bereitete Heiltränke. Ihm ging es vornehmlich um die Herstellung einer Arznei, die in Vergiftungsfällen ein wirksames Gegenmittel abgeben sollte. Das erzherzogliche Erzeugnis wurde auch an befreundete Fürsten abgegeben.

Ein gleich gerichtetes medizinisches Interesse bekundete auch Philippines Mutter, Frau Anna Welser, die Dr. Handsch in seinen Aufzeichnungen mit Vorliebe die alte Welserin (vetula WeLSER) nennt. Sie hatte ihren Sitz am jenseitigen Innufer in der kleinen Weiherburg, weilte jedoch zumeist bei Tochter und Enkeln in Ambras. Die alte Freundin der Arzneikunst besaß einen guten Humor, wie einer ihrer Aussprüche bezeugen mag: «Es ist nicht müglich, daß ein Altes immer gesundt sei. Alte Wagen krachen gern.» Noch als 65jährige steigt Frau AnNa ins Gebirge hinan, um dem Schrei der Hirsche zu lauschen.

Den kleinen Kreis der Heilkundler auf Ambras schloß die Tante Loxan, die über das Liebesverhältnis Philippines und Frrdinands am besten unterrichtet und ihrer Nichte aus Böhmen nach Ambras gefolgt war. Auch Frau Loxan hat nachweislich ein Arzneibuch geführt, das die Aufmerksamkeit des Dr. HandscH erregte. Die Rezepte der Tante müssen selbst für den Fachmann beachtenswert gewesen sein, sonst hätte er sich wohl nicht die Mühe genommen, einen guten Teil ihres Buches abzuschreiben und einem seiner medizinischen Kompendien einzuverleiben.

Die Führung auf dem Gebiete der Hausmedizin lag jedoch bei Philippine. Wenn eines der Familienmitglieder erkrankte, war sie zur Stelle und schöpfte sie aus dem Schatz ihrer medizinischen Erfahrungen. Am häufigsten war dies bei ihrem Gemahl notwendig, dessen Befinden durch sein Steinleiden (Nierensteine) und andere Erkrankungen oft gestört war. Philippine war dem Erzherzog die beste «Aufwarterin», wie dieser selbst gelegentlich hervorhob.

Die eigenen Leiden PhILIPpines spiegeln sich wohl in der größeren Zahl von Rezepten wider, die gerade für Steinleiden - bei Philippine waren es Gallensteine - und für die «Mutter», d. i. Gebärmutter, verzeichnet sind. Von den Schmerzen, die sich im letzten Lebensjahrzehnt einstellten, meinte Philippine einmal, es wäre ihr, als drückten Himmel und Erde auf sie. Und ein anderes Mal, es würden hundert Messer in sie dringen.

Am stärksten vertreten sind jedoch die Rezepte, die sich mit den Kinderkrankheiten befassen. Für ein und dieselbe Krankheit sind hier häufig mehrere Rezepte gebucht. Gewiß spricht da die Sorge der Mutter um die eigenen Kinder mit. Auch an Ratschlägen für schwangere Frauen fehlt es nicht; sie reichen bis zum Geburtsakt hin. Die Kinderliebe PhilipPines kommt auch in Wortgebrauch und 
Sprache ihrer Niederschriften zu schönem Ausdruck. Da gibt es nur ein : bäuchli, mindly, ein megly, herzy, herzgrübly usw.

Aus mehr als einer Stelle ist zu ersehen, wie sehr Philippine Gewicht darauf legte, daß nicht erst gewartet werde, bis sich eine Krankheit einstellt, sondern daß sie möglichst verhütet werden soll. Mit solcher «Fürsehung» entspricht Philippine den in der medizinischen Literatur ihrer Zeit oft anzutreffenden Gesundheitsregeln (regimen sanitatis). Die zahlreichen Rezepte für Gurgelwässer und für Zahnpflege sind jedenfalls unter dem Gesichtspunkt der Vorbeugung zu verstehen. Neben der Sorge, die Zähne «weiß und frisch» zu erhalten, steht die Meinung Philippines, daß es das beste sei, den schlechten Zahn beizeiten «auszubrechen», denn «einer verderpt den andern».

Philippine gibt auch Hinweise, woran die betreffende Krankheit zu erkennen wäre, wirft ab und zu auch die Frage nach den Ursachen auf. Fraisen der Kinder z.B. will sie darauf zurückführen, daß die Kinder erschreckt wurden oder auf den Zorn der Amme oder auch der Kinder selbst.

Mehr Raum widmet PhiLıppine der «bösen Krankheit», der Pestilenz, die vor und nach 1570 das Land heimsuchte. Die Schrecknisse dieser Krankheit hatte Philippine bereits 1562 in Böhmen miterlebt. Man möge, so rät Philippine, wenn die Gefahr droht, den Genuß gewisser Speisen - sie werden aufgezählt - meiden. Fällt die Krankheit doch ein, dann wären die von ihr verzeichneten Arzneien in bestimmter Abfolge zu nehmen. Die noch nicht Erkrankten sollen sich vor dem «Dampf» der schwitzenden Kranken hüten und sich in möglichst großem Abstand halten, daß niemand vergiftet werde. Bett und Bettgewand sollen gesondert gelegt und gewaschen werden und lange an der Luft hängen. Wer zum kranken Menschen gehen will, der soll zuvor von einer bestimmten Latwerge in der Größe einer halben Haselnuß essen.

Viele der Krankheitsbezeichnungen sind uns heute fremd, außer der Leser des Rezeptbuches wäre Kenner der Geschichte der Pharmazie. Wer erkennt z. B. firicht als Krampf, flisch als Rheuma, undeuen als Erbrechen, freset sur als Krätzmilbe, roßmuggen als Sommersprossen, usw. ?

In den Jahren 1571 und 1574 suchten der Erzherzog und Philippine mit Gefolge Karlsbad auf, um eine Behebung oder Linderung ihrer Steinleiden zu erreichen. Dr. HANDSGH begleitete sie und machte in seinen Diarien genaue Aufzeichnungen über den Verlauf der Kur. Diese fiel etwas massiv aus. Neben dem Trinken des Sprudels und dem Gebrauch der Bäder spielten auch Mittel zum Schweißtreiben und Abführen eine Rolle. Ein durchgreifender Erfolg war bei unseren Gästen nicht zu verzeichnen, wohl aber hat die wiederholte Anwesenheit des hohen Paares den Ruf des alten Kurortes und seiner Heilwässer nicht wenig erhöht.

Nicht sonderlich wundernehmen kann es, daß Philippine, wie viele Zeitgenossen, auch Ärzte, im Banne von allerlei abergläubischen Vorstellungen stand. 
Phillppine ist in dieser Hinsicht ganz Kind ihrer Zeit. Da wird z.B. «die hitz» des Kranken dadurch bekämpft, daß neunerlei Eisen ins Krankenbett gelegt werden. Oder wenn ein Kind das Reißen im Bauch hat, so kann ihm geholfen werden, wenn man neunerlei Holz in die Wiege gibt. Beim Auftreten von Würmern kann Abhilfe geschaffen werden, wenn eine Attichwurzel, die im Monat August bei abnehmendem Mond gesucht wurde, um den Hals gehängt wird.

Schließlich empfiehlt die Rezeptensammlung auch Mittel besonderer Art, über die der Mensch des 20. Jahrhunderts vielleicht ein wenig den Kopf schütteln mag. Dabei ist in erster Linie an die Verwendung des menschlichen Harns gedacht. Um die Gesundung von Kindern zu erreichen, wird u. a. der Harn der Mutter oder der des Vaters und der Mutter herangezogen. Wenn heute noch in der ländlichen Bevölkerung der Glaube an die Heilkraft des Harns zu treffen ist, so wird dieser Glaube im 16. Jahrhundert nicht allzusehr auffallen können. Geschätzt ist auch der weiße Hühnerkot. Das eine Mal ist er Bestandteil bei Bereitung einer Brandsalbe, ein anderes Mal - bei Kolikbekämpfung - wird er mit einem Körnchen Bernstein verrieben, in Rotwein gekocht und getrunken.

Ein schönes Zeugnis wird PhILIPpine ausgestellt durch ihren Appell an den Krankenpfleger, «fleiß zu haben», und ebenso durch ihre Mahnung an die Mutter zur Geduld: «da mußts eine gute weyl versuchen und nit flux aufhern, wenn es nit von stund an hilft.»

Das Rezeptbuch ist nicht zuletzt auch ein beredtes Zeugnis für den frommen, religiösen Sinn seiner Besitzerin. Die Belege hiefür sehen wir in Wendungen wie: «da got der allmächtige ein jedes mensch davor behuet», "got allein die er», «ist mit gotes hilf leichtlich zu wenden», «got dem allmächtigen sey lob, der geb weiter sein gnad, Amen» u. ä. Mit solchem Gottvertrauen und Dank gegen Gott stellte sich die Schloßherrin an die Seite ihres Leibarztes, der an die Spitze eines seiner Kompendien u. a. die Worte schrieb: «Arznei hilft, so gott will.»

Hauptquelle für diese Skizze war Philippines Rezeptbuch. Aus ihm kann reichlich schöpfen jeder, der sich mit der Geschichte des Arzneiwesens befaßt, aber auch der Volkskundler und Sprachforscher.

Die Nationalbibliothek in Wien verwahrt noch eine zweite von PhILIPpIne stammende Handschrift, ihr Kochbuch (Nr. 11.375). Dieses ist der Rezeptensammlung schon aus dem Grunde verwandt, weil auf seinen ersten Seiten Rezepte stehen, die weniger kulinarischer als medizinischer Art sind. Die Fülle der köstlichen Speisen, die in Gruppen zusammengestellt sind (Torten, Pasteten, Fastenspeisen usw.), gibt einen trefflichen Einblick in die Kochkunst an deutschen Fürstenhöfen, bevor die französische Küche ihren Einzug hielt.

Eine weitere Quelle für diese Ausführungen war das reiche handschriftliche Material, das auf den bereits mehrfach erwähnten Dr. Georg Handsch von Limus - Adel und Prädikat von Limus sind von Kaiser Ferdinand I. verliehen worden - zurückgeht und in der gleichen Bibliothek liegt. HANDSCH, der aus 
der deutschböhmischen Stadt Böhmisch-Leipa stammte und durch nicht weniger als 24 Jahre im Dienste des Erzherzogs Ferdinand stand, war ein Mann von vielseitigem Interesse. Er war Mediziner, Dichter, Geschichts- und Naturforscher und - ein Vielschreiber. Seine Aufzeichnungen, die dem medizinischen Gebiete zugehören, füllen Tausende von Seiten. Es sind meist Krankengeschichten. Eingestreut sind Hinweise auf den gesundheitlichen Zustand des Erzherzogs, PHILIPPINES, ihrer Kinder und Verwandten, aber auch auf ihren Charakter und ihr Wesen.

Bevor Handsch von Ambras Abschied nahm, überließ er seine Handschriften und Bücher gegen einen bescheidenen Geldbetrag dem Erzherzog. Sie gingen damit in die Ambraser Sammlung ein, die 1805 nach Wien kam. In seine Heimat zurückgekehrt, ist HANDSCH nach wenigen Monaten verstorben (1578).

Die Schloßherrin von Ambras ist, erst 53 Jahre zählend, ihrem Arzt bald im Tode nachgefolgt (1580). In allem, was über PhILIPPINe geschrieben wurde, steht der Preis ihrer Schönheit und die daran sich entzündende Liebe des jungen Habsburgers im Vordergrund. Philippine war und blieb die «bella Filipina», wie sie Erzbischof Granvella, der bekannte Staatsmann KarLS V., bewundernd genannt hatte.

Mehr noch wollen der edle Charakter und die hohen weiblichen Tugenden Philippines hervorgehoben sein: ihr mustergültiges, stilles, hausfrauliches Wirken, ihre durch nichts zu erschütternde Liebe zu ihrem Gemahl, ihre Herzensgüte und ihre nimmermüde Hilfsbereitschaft gegenüber ihren Mitmenschen. Diese Tugenden trugen PhiLippine die Liebe und Wertschätzung des ganzen Landes ein, und es war sicher keine bloße Redensart, wenn man bei Philippines Ableben im Lande hören konnte: «Wir haben an unserer gnädigsten Frau sehr übel verloren.»

\section{Literatur}

W. Bоенегм, Philippine Welser, eine Schilderung ihres Lebens und Charakters. Innsbruck 1894.

J. HrRn, Erzherzog Ferdinand II. von Tirol. Geschichte seiner Regierung und seiner Länder. 2 Bände. Innsbruck 1885 und 1888. 